\title{
Protocolo combinado de PEV y ERG en perros beagle para evaluar la integridad funcional de las vías visuales
}

\author{
Combined VEP and ERG protocol in beagle dogs to assess visual pathway functionality \\ D Torres-Soriano*, MC Tovar-Sahuquillo
}

Departamento de Medicina y Cirugía Animal, Facultad de Veterinaria, Universidad de Murcia, Murcia, España.

\begin{abstract}
SUMMARY
Visual evoked potentials (VEP) and electroretinography (ERG) are known as objective and quantitative methods to analyse the functionality of visual pathways. Separately used in the ophthalmologic clinic to evaluate retina (ERG) or central visual pathways (VEP), this study designs a combined protocol with both techniques recorded in one session, and also proposes a normal data base for beagle. Twelve beagle dogs were used to design a 90-minutes protocol by using a mydriatic drug, anaesthetic protocol and a proper electrode placement. Flash stimulation technique was selected, and monocular stimulations were recorded. A large number of parameters regarding light stimulation, recording, amplification, and signal averaging were also controlled. The sequence of testing was: VEP, ERG scotopic and ERG photopic. Dogs were dark-adapted for at least 20 minutes to record VEP and scotopic ERG, and evaluated in a darkened room. Photopic ERG was tested with a prior 10 minutes light-adaptation, in an illuminated room. Characteristic waveforms were obtained. Four peaks were present and named as: P1, N1, P2, and N2 for VEP recordings. However, in ERG recordings, typical a-wave and b-wave were registered, but c-wave was not present. The proposed electrodiagnostic combined protocol in this study allows the assessment of the visual pathway integrity from retina to visual cortex in dogs. This procedure allows to collect the information in the most simple, shorter and less stressful manner for the patient.
\end{abstract}

Key words: electrodiagnosis, electroretinography, visual evoked potentials, beagle.

\section{RESUMEN}

Los potenciales evocados visuales (PEV) y la electrorretinografía (ERG) son dos métodos de análisis objetivo y cuantitativo de la funcionalidad de las vías visuales, que se realizan de forma independiente en la clínica oftalmológica para la evaluación de la retina o de las vías visuales centrales, respectivamente. El presente trabajo propone un protocolo clínico combinado de ambas técnicas en una única sesión, además de una base de datos normales para la raza beagle. Se emplearon 12 beagles para diseñar un protocolo de 90 minutos de duración que incluía el uso de midriáticos, protocolo anestésico y colocación apropiada de electrodos de registro. La técnica de estimulación luminosa seleccionada fueron flashes de luz blanca emitidos de modo monocular. Diversos parámetros de estimulación, registro, amplificación y filtrado fueron calibrados. La secuencia de estimulación fue: PEV, ERG escotópico y ERG fotópico. Las 2 primeras técnicas se realizaron en oscuridad con previa adaptación ocular de 20 minutos, mientras que la última se realizó con luminosidad ambiente y previa adaptación a la luz de 10 minutos. La gráfica de PEV mostró 4 picos (P1, N1, P2, N2), mientras que el ERG mostró las típicas ondas (a y b) sin presencia de onda-c. Este procedimiento permite recoger los registros de ambas pruebas electrodiagnósticas de la manera más simple y menos estresante para el paciente canino, y propone una base de datos normales actualizada para el beagle.

Palabras clave: electrodiagnóstico, electrorretinografía, potenciales evocados visuales, beagle.

\section{INTRODUCCIÓN}

La evaluación electrodiagnóstica proporciona una herramienta complementaria al examen clínico y neurológico, para evaluar la integridad funcional del sistema visual. La electrorretinografía (ERG) y los Potenciales Evocados Visuales (PEV) son las técnicas electrofisiológicas de mayor utilidad en oftalmología veterinaria. Se trata de técnicas objetivas, de gran sensibilidad y especificidad anatómica, que permiten el registro cuantitativo de los cambios de potencial eléctrico de las células reti-

Aceptado: 07.11.2013

* C/ Felix Marti Alpera, 50, $7^{\circ}$ A, Cartagena 30204, Murcia, España; denisets7@hotmail.com nianas y de las vías visuales centrales respectivamente, como respuesta a un estímulo luminoso de intensidad y frecuencia específica (Steiss 2003, Zaragoza 2011). Además, informan acerca del estado funcional de la vía visual, la posible localización de la afección y la evolución de la misma o del tratamiento instaurado.

En la clínica diaria, estas técnicas (fundamentalmente el ERG) permiten la detección precoz de alteraciones funcionales con anterioridad a su manifestación clínica. Además, el empleo de flashes como estímulos luminosos (técnica flash) es de gran utilidad en pacientes con opacidades de los medios transparentes del ojo y en procedimientos que requieran anestesia general (muy frecuente en oftalmología veterinaria para la inmovilización del animal) (Zaragoza 2011). De este modo, la frecuente ca- 
suística de cataratas acompañadas con atrofia progresiva de retina (APR) en caninos requiere del uso de esta técnica de estimulación de ERG para evaluar la actividad de los fotorreceptores, así como la evolución de dicha patología. Adicionalmente, los PEV complementan el estudio de la vía visual. Son de gran utilidad en pacientes con traumatismos craneoencefálicos donde la presencia de signos inflamatorios dificulta la evaluación de los reflejos pupilares fotomotores o de agudeza visual, así como también ocurre en neuropatías ópticas bilaterales. Teniendo en cuenta la imposibilidad de evaluación de las células ganglionares retinianas mediante el ERG con flashes de estimulación, los PEV resultan de gran ayuda en la evaluación indirecta de las mismas en estadios avanzados de glaucoma. No obstante, la detección precoz de glaucoma requiere del análisis con tomografía de coherencia óptica (TCO). Ambas técnicas también son de gran utilidad en animales de laboratorio como métodos de evaluación de la toxicidad de ciertos fármacos sobre la vía visual (Sato 1982).

A lo largo de estos años, varios investigadores han publicado estudios individualizados de ERG o de PEV en caninos (Sims y col 1989, Strain y col 1990, Narfström y col 1995, Narfström y col 2002, Dos Santos y col 2004), pero tan sólo Sato y col (1982) empleó ambas técnicas combinadas. Sin embargo, dicho estudio combinado (muy útil experimentalmente) resulta excesivamente invasivo (electrodos implantados en el hueso craneal) para realizar en la clínica ordinaria.

Durante estos años, se han establecido valores normales de ERG en humanos (Fishman y col 2001) y recientemente se han propuesto para perros Shih Tzu (Lee y col 2009), pero no hay constancia de bases de datos normales completas para beagles (Rosolen y col 2002 publicaron datos de ERG escotópico 0,01 y ERG fotópico 3,0). Respecto a los PEV, Strain y col (1990) publicó una serie de valores normales para el perro beagle empleando un protocolo individualizado de registro. De este modo, uno de los objetivos ha sido diseñar un protocolo clínico combinado de registro de ERG y PEV en caninos, que pueda ser realizado del modo más rápido y sencillo posible tanto en la clínica veterinaria como en el laboratorio experimental. Como objetivo adicional se pretendió proponer una base de datos normales para registros combinados de ambas pruebas en el perro de raza beagle.

En último lugar es necesario señalar la presencia de ciertas limitaciones al realizar dichas técnicas electrofisiológicas en la clínica diaria, como son: el coste del equipo de electrodiagnóstico así como su compleja calibración, la necesidad de cualificación específica del veterinario clínico para realizar una correcta interpretación de los registros, y la inexistencia de especificidad etiológica de los resultados que hace necesario el empleo de técnicas complementarias para hallar la causa de la disfunción. Aún así, la calidad diagnóstica que ofrecen estas técnicas es merecedora de su uso clínico y experimental.

\section{MATERIAL Y MÉTODOS}

\section{ANIMALES}

Se seleccionaron 12 perros beagle (11 machos y 1 hembra) de edades comprendidas entre los 2 y 5 años y con un peso corporal comprendido entre los 10 y los 21 $\mathrm{kg}$. Todos los animales pertenecían al Servicio de Animales de Laboratorio de la Universidad de Murcia (España) (Servicio de Apoyo a la Investigación-SAI, n REGA IS300305440012), siendo el estudio realizado siguiendo las directrices de la Unión Europea concernientes a la protección de animales empleados en experimentación (86/609/EEC).

Los animales fueron sometidos a un examen general, análisis de sangre y una exploración oftalmológica completa (test de respuesta a la amenaza, reflejos pupilares fotomotores, reflejo palpebral y corneal, respuesta al deslumbramiento, test de Schirmer, evaluación de la presión intraocular, examen con lámpara de hendidura, gonioscopia y oftalmoscopia directa e indirecta) con $24 \mathrm{~h}$ de antelación al estudio.

\section{PREPARACIÓN DE LA SALA DE ELECTRODIAGNÓSTICO}

Todos los tests se realizaron en una sala aislada de ruidos, con temperatura y luminosidad ambiente controlable y toma de oxígeno.

\section{PREPARACIÓN DEL PACIENTE}

Se diseñó un protocolo de sedación-anestesia que permitiera la relajación muscular del paciente con objeto de evitar el registro de artefactos derivados de la actividad eléctrica muscular (Narfström y col 2002). Para ello se inyectó intramuscularmente una combinación de medetomidina clorhidrato $(0,01 \mathrm{mg} / \mathrm{kg}$ p.v. $)$ y butorfanol tartrato (0,3 mg/kg p.v.) como sedantes. A continuación se colocó al paciente sobre la mesa de exploración en decúbito esternal manteniendo su cabeza apoyada horizontalmente sobre una colchoneta. Finalmente, el protocolo anestésico se completó inyectando intravenosamente una única dosis (5 mg/kg p.v.) de ketamina clorhidrato $(50 \mathrm{mg} / \mathrm{ml}$ de concentración). Así mismo, se le proporcionó oxígeno y calor corporal (mantas de calor) y se monitorizaron sus constantes vitales durante todo el protocolo diagnóstico.

Previamente a la estimulación luminosa, se instilaron gotas de tropicamida (1\%) cada $5 \mathrm{~min}$ durante $15 \mathrm{~min}$ para obtener una dilatación pupilar completa. Durante este proceso, se fue adaptando al paciente a la oscuridad ambiente durante un mínimo de $20 \mathrm{~min}$, ya que se seleccionó una secuencia de estimulación iniciada con las técnicas escotópicas (ausencia de luz ambiente) y finalizada con las fotópicas (presencia de luz ambiente) del siguiente modo: PEV (ambiente escotópico), ERG escotópico de 
$0,01 \mathrm{~cd} \cdot \mathrm{s} \cdot \mathrm{m}^{-2}$ (respuesta de bastones), ERG escotópico de $3,0 \mathrm{~cd} \cdot \mathrm{s} \cdot \mathrm{m}^{-2}$ (respuesta de conos y bastones), ERG fotópico de $3,0 \mathrm{~cd} \cdot \mathrm{s} \cdot \mathrm{m}^{-2}$ (respuesta de conos) y ERG flícker fotópico de $30 \mathrm{~Hz}$ y $3,0 \mathrm{~cd} \cdot \mathrm{s} \cdot \mathrm{m}^{-2}$ (elevada frecuencia de estimulación para obtener respuestas derivadas de las células localizadas exclusivamente tras el sistema de conos, inhibiendo las pertenecientes al sistema de bastones). El motivo de esta secuencia fue el máximo aprovechamiento de la adaptación a la oscuridad, ya que los bastones requieren mayor tiempo de adaptación a la oscuridad que los conos a la luz (tan sólo $10 \mathrm{~min}$ ). De este modo, el tiempo requerido de anestesia también fue menor.

\section{COLOCACIÓN DE ELECTRODOS}

En ambas técnicas se emplearon 3 electrodos: uno activo o de registro (+) próximo al área generadora de las respuestas eléctricas, otro de referencia (-) alejado del anterior, y otro de tierra (G) para completar el circuito eléctrico. Se utilizaron electrodos monopolares de superficie discoidales fijados a la superficie cutánea mediante una pasta conductora (TEN 20® conductive EEG paste, Weaver and Company, Aurora, Colorado, USA), por lo que fue necesario rasurar, limpiar y desinfectar con alcohol las áreas cutáneas de colocación. De este modo se disminuyó la resistencia al paso eléctrico entre la piel y el electrodo, detectándose por tanto registros sin artefactos o interferencias.

Para registrar los PEV, se adaptó a los caninos el sistema internacional 10/20 de colocación de electrodos para humanos (Odom y col 2010). Así, el electrodo de registro se fijó en la línea media de la protuberancia occipital, el de referencia en la línea media del hueso frontal entre ambos ojos, y el de tierra sobre la línea media del vértex entre ambas orejas.

Para el ERG, se utilizó el mismo tipo de electrodo de disco como electrodo de referencia situado $1 \mathrm{~cm}$ caudal al canto lateral del ojo a estimular y de tierra emplazado sobre la línea media del hueso occipital. Sin embargo, se empleó un electrodo de lente de contacto (ERGjet ${ }^{\circledR}$ electrode, Roland Consult, Brandenburg, Germany) como electrodo activo de registro. Éste se colocó sobre la córnea con un gel de carbómero (conductor eléctrico y protector de la superficie corneal) evitando la formación de burbujas entre el electrodo y la córnea, puesto que podrían causar artefactos. Adicionalmente, para mantener el ojo abierto, evitando el parpadeo causante del desplazamiento del electrodo y del registro de artefactos, se realizaron dos puntos en "U" con una sutura monofilamento del $n^{\circ} 0$ en la conjuntiva bulbar a las 12 y a las 6 respectivamente.

\section{ORGANIZACIÓN DEL EQUIPO}

El equipo de electrodiagnóstico empleado en este estudio fue RETIsystem ${ }^{\circledR}$ (Roland Consult, Germany) con el programa de análisis RETIport 32 para registrar PEV y ERG.
En ambas técnicas, los electrodos se conectaron a un amplificador diferencial de la señal de 1 canal, y éste, a su vez, a la unidad informática central del equipo. Finalmente, un mini-ganzfeld portátil utilizado como fotoestimulador monocular también fue conectado a la unidad central.

\section{CALIBRACIÓN DEL SISTEMA}

Con antelación a la estimulación luminosa, se seleccionó el tipo de técnica flash para PEV y ERG recomendado por la Sociedad Internacional de Electrofisiología Clínica de la Visión (ISCEV) para pacientes anestesiados o con opacidades de medios transparentes del ojo (otras técnicas con patrones luminosos requieren de la participación activa del paciente). A continuación, fue necesario calibrar minuciosamente diversos parámetros de estimulación y registro en relación a la técnica seleccionada. Adaptando a los caninos las directrices de la ISCEV (Marmor y col 2009, Odom y col 2010), se han recopilado dichos parámetros a controlar en el cuadro 1 .

\section{ESTIMULACIÓN LUMINOSA}

En primer lugar, fue de gran importancia controlar la luminosidad ambiental para cada técnica, así los PEV, ERG escotópico 0,01 y ERG escotópico 3,0 se realizaron en oscuridad ambiente (además de la adaptación a la oscuridad previa ya comentada en la preparación del paciente), mientras que el ERG fotópico 3,0 y el ERG flícker fotópico de $30 \mathrm{~Hz}$ se realizaron bajo luminosidad ambiente (además de 10 min de adaptación previa a la luz).

Como estímulos luminosos, se emplearon flashes de luz blanca emitidos por un mini-ganzfeld portátil colocado próximo al ojo a estimular sin tocar los párpados. Se realizaron estimulaciones monoculares (empezando sistemáticamente por el ojo derecho) tapando el ojo adelfo con un parche opaco para aislar las respuestas de cada ojo y poder, por tanto, detectar asimetrías funcionales entre ambos ojos. Para cada técnica, se realizaron 2 estimulaciones completas en cada ojo ( 2 tests en ojo derecho seguidos de 2 tests en ojo izquierdo) en los 12 beagles, por lo que se obtuvieron 48 registros en cada tipo de prueba. Los dos registros consecutivos en cada ojo permitieron confirmar la reproducibilidad de las respuestas.

\section{SISTEMA DE REGISTRO Y PROMEDIADO}

El electrodo activo permitió el registro de señales evocadas por la corteza visual (PEV) y por las células de la retina (ERG) como respuestas a los estímulos luminosos. Sin embargo, para analizar las diminutas señales procedentes del sistema visual fue necesario amplificarlas y filtrarlas (ver ajuste de filtros en cuadro 1) para discernirlas de la actividad electroencefalográfica y muscular. Dichas señales filtradas se enviaron a continuación a la unidad central para ser promediadas y representadas a modo de gráficas. 
Cuadro 1. Parámetros PEV y ERG de estimulación y registro.

VEP and ERG stimulation and recording parameters.

\begin{tabular}{|c|c|c|c|c|c|}
\hline Parámetro & PEV & ERG esc 0,01 & ERG esc 3,0 & ERG fot 3,0 & ERG Flícker fot \\
\hline $\begin{array}{l}\text { Luminosidad } \\
\text { ambiental* }\end{array}$ & Escotópico & Escotópico & Escotópico & Fotópico & Fotópico \\
\hline Estimulación & Monocular & Monocular & Monocular & Monocular & Monocular \\
\hline $\begin{array}{l}\text { Tiempo adaptación } \\
\text { oscuridad/luz (min) }\end{array}$ & 20 & 20 & 30 & 10 & 10 \\
\hline Distancia Ojo-miniganzfeld $(\mathrm{cm})$ & 5 & 5 & 5 & 5 & 5 \\
\hline Intensidad del Flash (cd. s. $\mathrm{m}^{-2}$ ) & 3 & 0,01 & 3 & 3 & 3 \\
\hline Luminancia de fondo $\left(\mathrm{cd} \cdot \mathrm{m}^{-2}\right)$ & 30 & Ninguna & Ninguna & 30 & 30 \\
\hline Frecuencia de estimulación (flash/s) & 1 & $\leq 0,5$ & 0,5 & $4,9-5,1$ & 30 \\
\hline Tiempo entre flashes (s) & Ninguno & 2 & 10 & $\geq 0,5$ & Ninguno \\
\hline Impedancia del electrodo $(\mathrm{K} \Omega)$ & $5-7$ & $2-5$ & $2-5$ & $2-5$ & $2-5$ \\
\hline Filtros $(-3 \mathrm{db})(\mathrm{Hz})$ & $1-100$ & $0,3-300$ & $0,3-300$ & $0,3-300$ & $0,3-300$ \\
\hline Tiempo de barrido (ms) & 250 & 250 & 250 & 200 & 200 \\
\hline $\mathrm{N}^{\mathrm{o}}$ barridos promediados & 64 & No necesario & No necesario & No necesario & No necesario \\
\hline
\end{tabular}

Adaptado de las recomendaciones de la ISCEV (Marmor y col 2009, Odom y col 2010).

Adapted from the ISCEV recommendations (Marmor et al 2009, Odom et al 2010).

* Escotópico = ausencia de luz ambiental. Fotópico = presencia de luz ambiental.

\section{ANÁLISIS ESTADÍSTICO E INTERPRETACIÓN DE RESULTADOS}

El conjunto de gráficas derivadas de cada técnica fue registrado en un formulario de electrodiagnóstico individual para cada beagle examinado. Cada gráfica fue analizada estudiando su morfología general ( $\mathrm{n}^{\mathrm{o}}$ de ondas (ERG) o picos (PEV), su polaridad, su secuencia de aparición y el tamaño de las mismas). Siguiendo las recomendaciones de la Sociedad Española de Oftalmología (SEO), consideraríamos que un registro es patológico cuando la morfología de la gráfica muestra alteraciones significativas de 2 ó más de estos parámetros registrados repetidamente en un individuo (Zaragoza 2011).

Como datos cuantitativos, se registraron y analizaron los tiempos implícitos (TI) (también conocidos como latencias absolutas) medidos en ms y las amplitudes (Amp) medidas en $\mu \mathrm{V}$ de los picos de cada onda representada. En ambas técnicas, los tiempos implícitos indican el tiempo que tarda un conjunto celular en presentar su respuesta máxima tras el estímulo luminoso, mientras que las amplitudes muestran la intensidad de dichas respuestas (Zaragoza 2011).

Para finalizar el estudio, se realizó el análisis estadístico descriptivo de los datos obtenidos mediante el programa específico SPSS Statistics 12 (IBM Corporation, Somers, NY). Se recopilaron los datos de medias y desviaciones estándar en los cuadros 2 y 3.

\section{RESULTADOS}

\section{GRÁFICAS}

Los registros de PEV mostraron gráficas típicas con morfología en 'M' (figura 1a-b) compuestas por 4 picos secuenciados del siguiente modo: P1, N1, P2 y N2. Esta nomenclatura es acorde a la polaridad $(\mathrm{P}=$ positivo, $\mathrm{N}=$ negativo) y al orden de aparición de los picos. En todos los registros, $\mathrm{P} 2$ fue el pico más prominente. Sin embargo, no obtuvimos ningún pico $\mathrm{P} 3$ consistente en nuestro estudio.

Los ERG fueron analizados individualmente según el tipo de estimulación. En primer lugar, los ERG escotópicos $0,01\left(\mathrm{~cd} \cdot \mathrm{s} \cdot \mathrm{m}^{-2}\right)$ mostraron gráficas compuestas por una única onda-b positiva resultante de la actividad de las células bipolares y de Müller derivada de la estimulación de los bastones (figura 1c). No se obtuvo ninguna onda-a $u$ onda-c.

Los ERG escotópicos 3,0 (cd. s· $\left.\mathrm{m}^{-2}\right)$ revelaron gráficas formadas por una onda-a negativa, representante de la actividad eléctrica de conos y bastones, seguida de una onda-b positiva de mayor intensidad, formada a partir de la actividad de células bipolares y de Müller derivada de la estimulación de ambos tipos de fotorreceptores (figura 1d). En comparación con el ERG escotópico 0,01 anterior, esta onda-b presentó menor TI y mayor Amp.

Las gráficas del ERG fotópico 3,0 (cd· s $\left.\mathrm{m}^{-2}\right)$ también estaban compuestas por las típicas ondas-a y b (figura 1e), 
Cuadro 2. PEV: datos estadísticos descriptivos de tiempos implícitos y amplitudes.

VEP: peak times and amplitudes descriptive statistical data.

\begin{tabular}{lcccccc}
\hline & & \multicolumn{2}{c}{ Tiempos implícitos $(\mathrm{ms})$} & \multicolumn{2}{c}{ Amplitudes $(\mu \mathrm{V})$} \\
\hline Media & P1 & N1 & P2 & N2 & P1N1 & P2N2 \\
DE & 16,9 & 36,3 & 91,7 & 142,37 & 2,28 & 1,54 \\
\hline
\end{tabular}

Todos los datos han sido calculados a partir de dos exploraciones en cada ojo de 12 beagle $(n=48)$.

All data have been calculated with two explorations in both eyes of 12 beagle dogs $(n=48)$.

Cuadro 3. ERG: datos estadísticos descriptivos (media \pm DE) de tiempos implícitos y amplitudes.

ERG: peak times and amplitudes descriptive statistical data (mean $\pm \mathrm{SD})$.

\begin{tabular}{|c|c|c|c|c|}
\hline & \multicolumn{2}{|c|}{ Onda-a } & \multicolumn{2}{|c|}{ Onda-b } \\
\hline & $\mathrm{TI}(\mathrm{ms})$ & $\operatorname{Amp}(\mu \mathrm{V})$ & TI (ms) & $\operatorname{Amp}(\mu \mathrm{V})$ \\
\hline ERG esc $0,01^{*}$ & ----------- & ------------ & $64,4 \pm 10,2$ & $86,5 \pm 51,4$ \\
\hline ERG esc 3,0 & $15,4 \pm 3,1$ & $56,2 \pm 28,1$ & $35,6 \pm 4,1$ & $171,5 \pm 80,7$ \\
\hline ERG fot 3,0 & $12,0 \pm 2,4$ & $6,4 \pm 4,6$ & $28,1 \pm 2,3$ & $37,7 \pm 21,6$ \\
\hline ERG Flicker $30 \mathrm{~Hz}$ fot ${ }^{* *}$ & ------------ & ------------- & $26,3 \pm 3,2$ & $36,3 \pm 27,8$ \\
\hline
\end{tabular}

Todos los datos han sido calculados a partir de dos exploraciones de ambos ojos en 12 perros beagle $(\mathrm{n}=48)$. TI = tiempo implícito. Amp = amplitud. Esc = escotópico (ninguna luminosidad ambiente ni luminosidad de fondo). Fot = fotópico (luminosidad de fondo de $30 \mathrm{~cd} \cdot \mathrm{m}^{-2} \mathrm{y}$ luminosidad ambiental).

* ERG esc 0,01 no muestra onda-a.

** ERG Flicker $30 \mathrm{~Hz}$ fot $\left(3,0 \mathrm{~cd} \cdot \mathrm{m}^{-2}\right)$ no muestra onda-a, solo onda-b (denominada P2 en la figura 1f).

All data have been calculated from two explorations in both eyes of 12 beagle dogs $(n=48)$.

$\mathrm{TI}=$ peak time. Amp $=$ amplitude. Esc. $=$ scotopic $($ no background luminance nor environment luminosity). Fot. $=$ photopic (background luminance of $30 \mathrm{~cd} \cdot \mathrm{m}^{-2}$ and environment luminosity).

* ERG esc 0,01 shows no a-wave.

** ERG Flicker $30 \mathrm{~Hz}$ fot $\left(3.0 \mathrm{~cd} . \mathrm{m}^{-2}\right)$ shows no a-wave, only b-wave (named as $\mathrm{P} 2$ in figure 1f).

derivadas de la estimulación del sistema de conos. Comparando con el ERG escotópico 3,0, esta prueba mostró ambas ondas de menor Amp, aunque de menor TI, es decir, las respuestas derivadas exclusivamente del sistema de conos fueron más rápidas pero menos intensas. Del mismo modo, se apreciaron las mismas diferencias al comparar la onda-b del ERG escotópico 0,01.

Finalizando el protocolo, el análisis morfológico de las gráficas del ERG flícker fotópico de $30 \mathrm{~Hz}$ mostró una única onda positiva de repetición rítmica a lo largo de todo el tiempo de análisis (figura 1f). Esta onda, registrada por nuestro sistema como P2, coincide con la onda-b descrita por ciertos autores (Ropstad y Narfström 2007) y representa la actividad celular post-receptoral (células retinianas situadas tras los fotoreceptores) derivada de la estimulación del sistema de conos.

En todos los registros se observó similitud entre las 2 gráficas obtenidas en un mismo ojo, así como entre las gráficas de ambos en un mismo beagle (ejemplo en figura 1a-b).
Además la comparación morfológica de gráficas entre los 12 beagles tampoco mostró diferencias significativas. Ningún pico u onda estuvo ausente o significativamente retardado, ni se obtuvieron respuestas de Amp exagerada.

\section{DATOS ESTADÍSTICOS}

Los TI y las Amp de los diferentes picos y ondas, expresados en medias y desviaciones estándar (DE), fueron recopilados en los cuadros 2 y 3 para los PEV y ERG respectivamente.

En general, se observaron mayores DE de las Amp en comparación con los TI en los registros del ERG. Así mismo, se apreció aumento de las DE de los picos y ondas más tardíos en aparición tanto en los PEV como en los ERG. A pesar de estas observaciones, las medias se consideraron representativas cuando $\mathrm{DE} \leq \mathrm{R} / 3$ (siendo $\mathrm{R}$ = rango de datos), por lo que todos los datos resultaron representativos. 


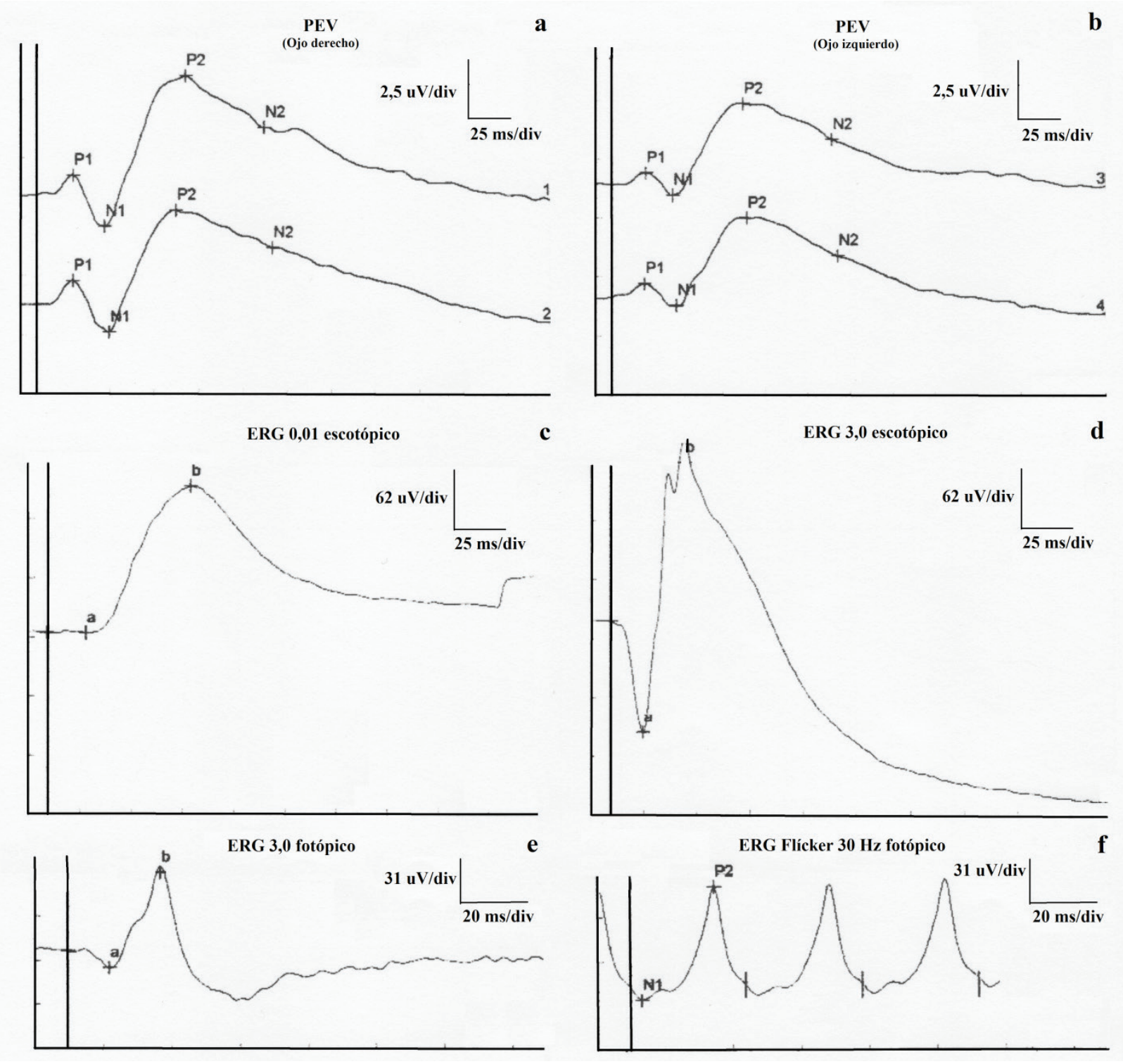

Figura 1. Gráficas representativas de cada prueba electrodiagnóstica: gráficas PEV de dos estimulaciones en ojo derecho (a) y ojo izquierdo (b), gráfica ERG 0,01 escotópico (c), gráfica ERG 3,0 escotópico (d), gráfica ERG 3,0 fotópico (e) y gráfica ERG Flícker $30 \mathrm{~Hz}$ fotópico (f).

Representative waveforms of each electrodiagnostic proof: VEP waveforms of two stimulations in right eye (a) and left eye (b), ERG 0.01 scotopic waveform (c), ERG 3.0 scotopic waveform (d), ERG 3.0 photopic waveform (e), and ERG Flícker 30Hz photopic waveform (f).

\section{DISCUSIÓN}

En este estudio se han incluido los PEV y ERG como técnicas electrodiagnósticas de mayor utilidad en la clínica oftalmológica veterinaria, así como en el laboratorio de experimentación animal, para la evaluación de la integridad funcional prequiasmática de la vía visual (Odom y col 2010). Estas herramientas diagnósticas presentan diversas ventajas como: la objetividad de los resultados, la posibilidad de registro no invasivo, la relativa corta duración del protocolo (se obtiene gran información en tan sólo $50 \mathrm{~min}$ ) y la posibilidad de diagnóstico precoz que aportan con antelación a la manifestación clínica de la patología.

Normalmente, en la clínica diaria suele emplearse el ERG con flashes previamente a la cirugía de cataratas con intención de valorar la funcionalidad de la retina. Sin embargo, hay que tener en cuenta que esta técnica es incapaz de valorar las células ganglionares y el resto de la vía visual. Por tanto, el presente protocolo combinado de ERG y PEV pretende completar el estudio de la vía visual, evaluando indirectamente la funcionalidad de las células ganglionares y del resto de la vía visual hasta la corteza visual mediante los PEV. En concreto, nuestro 
registro de PEV mediante un único electrodo occipital permite la evaluación de la zona prequiasmática, mientras que según la SEO (Zaragoza 2011) serían necesarios electrodos adicionales en ambos hemisferios cerebrales para concretar de modo más preciso la localización de la disfunción visual. Este protocolo informaría a cerca de la indicación de la cirugía de cataratas y mejoraría el pronóstico de la misma. No obstante, de manera complementaria, sería recomendable evaluar el sistema nervioso central (SNC), mediante exámenes neurológicos y resonancia magnética, del paciente que presente alteraciones de las pruebas electrodiagnósticas.

En la realización de este estudio se seleccionaron beagles adultos puesto que ciertos investigadores han probado la influencia de la edad en PEV (Strain y col 1991 ${ }^{\text {b }}$ Kimotsuki y col 2006, Odom y col 2010) y ERG (Qiu y col 2002) debido a la escasa mielinización y el reducido número de conexiones sinápticas en el SNC de neonatos y seniles. Así mismo, se seleccionó la raza beagle por su frecuencia de utilización como animal de experimentación.

A la hora de plantear una secuencia de técnicas diagnósticas para analizar la vía visual, lo más frecuente sería comenzar evaluando la retina (mediante ERG) para finalizar con el resto de la vía visual (mediante PEV). Sin embargo, este estudio comienza con el registro de los PEV (en ambiente escotópico) para continuar con los ERG escotópicos y finalizar con los ERG fotópicos. De este modo conseguimos reducir el tiempo total de análisis a $50 \mathrm{~min}$ y por tanto disminuir el tiempo de anestesia. Además, teniendo en cuenta la gran sensibilidad de los bastones a la estimulación luminosa, es aconsejable realizar las pruebas escotópicas con antelación a las fotópicas, de manera contraria sería necesario adaptar la retina a la oscuridad al menos durante $1 \mathrm{~h}$ para recuperar la funcionalidad de los bastones saturados (Marmor y col 2009, Odom y col 2010). De modo similar, el tiempo de adaptación previa a la oscuridad fue muy importante en este estudio. La gran abundancia de bastones en la retina canina, así como su mayor contribución en la corteza visual (Dos Santos y col 2004) hacen pensar en este tipo celular como el mayor contribuyente en los PEV, por lo que se decidió realizar esta técnica en oscuridad ambiental para detectar los cambios de luminancia requeridos por los bastones. Por tanto, fueron necesarios al menos 20 min de pre-adaptación a la oscuridad (aprovechados para realizar la sedación - anestesia y preparación del paciente) como recomienda la ISCEV (Marmor y col 2009, Odom y col 2010) para preparar los bastones y registrar respuestas más rápidas e intensas (Hyung-Ah y col 2007). Aunque Strain y col (1990) publicó un estudio sin preadaptación a la oscuridad, nuestras pruebas previas en dichas condiciones no mostraron registros de buena calidad. Finalmente, los ERG fotópicos tan sólo requirieron de 10 min de adaptación a la luz al igual que documentan otros autores (Marmor y col 2009, Narfström y col 2000, Narfström y col 2002, Safatle y col 2005).
A diferencia del uso generalizado de midriáticos en los protocolos de ERG (Zaragoza 2011), existe gran controversia en cuanto al uso de los mismos para obtener PEV. La ISCEV (Odom y col 2010) y la SEO (Zaragoza 2011) no consideran necesario el empleo de los mismos en humanos, pues la abundancia de conos en el área centralis de la retina humana y gran representación de los mismos en la corteza visual occipital tan sólo hace necesario estimular el área central de la retina. Sin embargo, la retina del perro solamente presenta un 5\% de conos (Curtis y Lightfoot 1993), lo que haría suponer la existencia de una mayor representación de los bastones en la corteza occipital canina (aunque no hay evidencia de publicaciones al respecto). En nuestro estudio fue necesario dilatar la pupila por completo para obtener respuestas de una cierta magnitud. Adicionalmente, el uso de tropicamida evitó la posible miosis causada por los flashes luminosos.

De acuerdo con la mayoría de estudios publicados en animales (Sato y col 1982, Sims y col 1989, Uzuka y col 1989, Narfström y col 2002, Margalit y col 2003, Dos Santos y col 2004, Pontes y col 2004, Kimotsuki y col 2005, Safatle y col 2005, Kimotsuki y col 2006, Lin y col 2009), la utilización de un protocolo anestésico fue fundamental para conseguir la relajación completa del paciente evitando así los artefactos eléctricos derivados de la actividad eléctrica muscular, de los movimientos oculares o del parpadeo. Para evitar estos últimos, se realizaron suturas conjuntivales (Narfström y col 2000, Narfström y col 2002), las cuales fueron suficientes ya que la ketamina estabilizó en gran parte la desviación ventromedial del ojo causada por la medetomidina (Lin y col 2009). Otros investigadores emplean bloqueantes musculares (Safatle y col 2005) o inyecciones retrobulbares de $\mathrm{NaCl}$ (Narfström y col 1995) más invasivos para emplearlos en la clínica diaria y, este último método podría influir sobre el SNC (Andersson y col 2004).

Los electrodos de aguja son comúnmente empleados en animales (Strain y col 1990, Strain y col 1991ª, Strain y col 1991 ${ }^{\text {b }}$, Narfström y col 1995, Damiani y col 1998, Perez-Salvador 1999, Narfström y col 2000, Komáromy y col 2002, Narfström y col 2002, Dos Santos y col 2004, Kimotsuki y col 2005, Kimotsuki y col 2006, Özkaya y col 2007, Hyung-Ah y col 2007), pero los electrodos de disco superficiales no son invasivos (eliminan el riesgo de infección cutánea) y proporcionan registros de buena calidad (sin interferencias). El ERGjet ${ }^{\circledR}$, de contacto para la córnea, recomendado por la ISCEV (Marmor y col 2009), proporciona registros muy estables y es asequible para usarlo habitualmente. Del mismo modo, posicionando el electrodo activo sobre la línea media del hueso occipital para registrar los PEV, se pudo detectar la zona de respuestas de mayor intensidad. Pruebas realizadas en zonas adyacentes no produjeron registros por problemas de impedancia de los electrodos. Se pudo comprobar, por tanto, que el sistema internacional 10/20 para el registro de PEV y ERG era perfectamente extrapolable a los caninos. 
Respecto al tipo de estimulación, varios autores (Uzuka y col 1989, Pérez-Cobo y col 1994, Damiani y col 1998, Odom y col 2010) prefieren realizar estimulaciones binoculares para evaluar de un modo global la visión del paciente. Por el contrario, en este estudio decidimos realizar estimulaciones monoculares para detectar posibles asimetrías funcionales entre ambos ojos o para asegurar la similitud de registros en un mismo perro y así elaborar una base de datos normales fiable y reproducible. Posiblemente, estimulando binocularmente, la actividad de un ojo podría compensar o enmascarar la disfunción del ojo adelfo.

En relación al calibrado del equipo de electrodiagnóstico, en este trabajo hemos podido emplear las recomendaciones de la ISCEV (Marmor y col 2009, Odom y col 2010), pero es fundamental advertir al clínico de la necesidad de ajustar el protocolo a cada equipo y a los requerimientos particulares de cada paciente.

Respecto a los resultados del presente trabajo, las gráficas de PEV mostraron morfología en 'M' similares a otros estudios en perros (Ropo y col 1992, Pérez-Cobo y col 1994, Margalit y col 2003, Odom y col 2010). La ISCEV (Odom y col 2010) identifica cuatro picos en PEV humanos con el siguiente orden de aparición: N1, P1, N2 y P2; sin embargo, recientes estudios en caninos (Strain y col 1990, Strain y col 1991 b, Margalit y col 2003, Kimotsuki y col 2005, Kimotsuki y col 2006) muestran una secuencia de picos como la nuestra: P1, N1, P2 y N2. Autores como Kimotsuki y col $(2005,2006)$ y Strain y col $\left(1990,1991^{\text {b }}\right)$ hallaron un quinto pico P3, pero este pico fue muy difícil de localizar en nuestros registros. La causa podría ser la diferencia entre equipos de electrodiagnóstico.

Los datos cuantitativos obtenidos de TI en los registros de PEV presentaron grandes similitudes con otros estudios individualizados en perros (Strain y col 1990, Kimotsuki y col 2005, Kimotsuki y col 2006), donde P1 y N1 fueron los picos con TI menos dispares, mientras que $\mathrm{P} 2$ y N2 mostraron mayor variabilidad aunque sin ser significativa. Esto podría demostrar la mayor influencia de artefactos eléctricos en los picos más tardíos. En casi todas las publicaciones revisadas (Sato y col 1982, Uzuka y col 1989, Perez-Salvador 1999), las amplitudes P1-N1 y P2N2 (intensidad de las respuestas) fueron considerados los parámetros más importantes a interpretar, pero estos datos suelen mostrar más variabilidad que los TI, y estos últimos nos informarían del tiempo de respuesta celular (indirectamente muestra el grado de mielinización de la vía visual y la presencia de una posible compresión en la misma). Nuestros datos de amplitud varían respecto a los publicados, y éstos varían a su vez entre ellos. Por lo que, para una correcta interpretación de los mismos es fundamental comparar las amplitudes de varios tests en un mismo ojo así como entre ambos ojos de un mismo paciente (Odom y col 2010). La ISCEV propone calificar como patológicos los registros de dos o más picos anormales repetitivos para un mismo animal en tests consecutivos (Zaragoza 2011).
Respecto a los ERG, los hallazgos más significativos fueron: la ausencia de onda-c en caninos (posiblemente debido a artefactos derivados de la actividad muscular residual tras la onda-b), la obtención de gráficas de morfología típica en un protocolo combinado de electrodiagnóstico y la propuesta de una base normal de datos para el perro beagle no publicada con anterioridad (ciertos autores muestran una base de datos menos completa (Rosolen y col 2002); así mismo, se demuestra la posibilidad de obtención del ERG de forma conjunta con los PEV del modo más sencillo y rápido de obtención sin influencia significativa entre ambas técnicas.

Para concluir es necesario recordar que el protocolo combinado propuesto proporciona un método relativamente corto, objetivo, asequible y útil en la clínica veterinaria y laboratorio experimental para evaluar la funcionalidad global de la vía visual, por lo que animamos a los clínicos a aplicar este protocolo (completando el análisis neurológico y oftalmológico) previamente a la cirugía de cataratas. Finalmente, sería aconsejable el uso de los $\mathrm{PEV}$ en pacientes con glaucoma avanzado cuando el empleo de la TCO no sea posible o en traumatismo craneoencefálico para analizar la funcionalidad de la vía visual.

\section{AGRADECIMIENTOS}

El presente estudio forma parte de la Tesis de Doctorado de la primera autora llevada a cabo en la Universidad de Murcia (España). Las autoras desean agradecer a Doña Susana Henarejos y Don Sergio Hernández su colaboración en el análisis estadístico y su excelente asistencia técnica.

\section{REFERENCIAS}

Acland GM. 1988. Diagnosis and differentiation of retinal diseases in small animals by electroretinography. Semin Vet Med Surg Small Anim 3, 15-27.

Andersson F, O Etard, P Denise, L Petit. 2004. Early visual evoked potentials are modulated by eye position in humans induced by whole body rotations. BMC Neurosci 5 , 35 .

Curtis R, RM Lightfoot. 1993. The canine fundus. In: PetersenJones SM, Crispin SM (eds). Manual of small animal ophthalmology. $1^{\text {st }}$ ed. British Small Animal Veterinary Association, London, UK.

Damiani S, C Anzorandia, C Viera, M Márquez. 1998. Sensibilidad de los Potenciales Evocados Visuales a maniobras quirúrgicas y anestésicas durante la aproximación transesfenoidal. Rev Cubana Oftalmol 11, 39-47.

Dos Santos C, A Pontes, LP Da Veiga, F Dorea, JL Laus. 2004. The organization of flash electroretinography unit in veterinary medicine. Cienc Rural 34, 1097-1104.

Fishman GA, DG Birch, GE Holder, MG Brigell. 2001. Electrophysiologic testing in disorders of the retina, optic nerve and visual pathway. The Foundation of the American Academy of Ophthalmology, San Francisco, USA.

Hyung-Ah Y, J Man-Bok, P Shin-Ae, K Won-Tae, K Se-Eun, C Je-Min, Y Na-Young, S Kang-Moon. 2007. The determi- 
nation of dark adaptation time using electroretinography in conscious Miniature Schnauzer dogs. J Vet Sci 8, 409414.

Kimotsuki T, M Yasuda, S Tamahara, N Matsuki, K Ono. 2005. Topographic analysis of Flash Visual Evoked Potentials in dogs. J Vet Med Sci 67, 869-875.

Kimotsuki T, M Yasuda, S Tamahara, M Tomihari, N Matsuki, K Ono. 2006. Age-associated changes of Flash Evoked Potentials in dogs. J Vet Med Sci 68, 79-82.

Komáromy AM, DE Brooks, WW Dawson, ME Källberg, FJ Ollivier, R Ofri. 2002. Technical issues in electrodiagnostic recording. Vet Ophthalmol 5, 85-91.

Lee JS, KH Kim, HY Jang, B Lee, JY Kim, SW Jeong. 2009. The normal electroretinogram in adult healthy Shih Tzu dogs using the HMsERG. $J$ Vet Sci 10, 233-238.

Lescure F. 1992. Electrophysiologie oculaire. In: Lescure F (ed). Encyclopédie Veterinaire, Ophtalmologie 1700. Editions Techniques, Paris, France, Pp 1-13.

Lin SL, WC Shiu, PC Liu, FP Cheng, YC Lin, WS Wang. 2009. The effects of different anesthetic agents on short electroretinography protocol in dogs. $J$ Vet Med Sci 71, 763-768.

Margalit E, JD Weiland, RE Clatterbuck, GY Fujii, M Maia, M Tameesh, G Torres, SA D'Anna, S Desai, DV Piyathaisere, A Olivi, E de Juan, MS Humayun. 2003. Visual and electrical evoked response recorded from subdural electrodes implanted above the visual cortex in normal dogs under two methods of anesthesia. J Neurosci Meth 123, 129-137.

Marmor MF, AB Fulton, GE Holder, Y Miyake, M Brigell, M Bach. 2009. ISCEV standard for full-field clinical electroretinography (2008 update). Doc Ophthalmol 118, 69-77.

Narfström K, BE Andersson, S Andreasson, P Gouras. 1995. Clinical electroretinography in the dog with ganzfeld stimulation: a practical method of examining rod and cone function. Doc Ophthalmol 90, 279-290.

Narfström K, B Ekesten, SG Rosolen, BM Spiess, CL Percicot, R Ofri. 2000. Recommendations for a harmonized ERG protocol. Proceedings of the $1^{\text {st }}$ European Conference on Veterinary Visual Electrophysiology, Vienna, Austria, Pp 21-25.

Narfström K, B Ekesten, SG Rosolen, BM Spiess, CL Percicot, R Ofri. 2002. Guidelines for clinical electroretinography in the dog. Doc Ophthalmol 105, 83-92.

Odom VJ, M Bach, C Barber, M Brigell, MF Marmor, AP Tormene, GE Holder. 2004. Visual evoked potentials standard (2004). Doc Ophthalmol 108, 115-123.

Özkaya YG, A Agar, G Hacioglu, P Yargiçoglu. 2007. Exercise improves visual deficits tested by visual evoked potentials in Streptozotocin-induced diabetic rats. Tohoku J Exp Med 213, 313-321.

Pérez-Cobo JC, M López De Armentia, S Sánchez-Suero, M Pérez-Arroyo. 1994. Visual Evoked Potentials in response to flashes in the cat cortex. Rev Esp Fisiol 50, 183-190.

Perez-Salvador E. 2003. Valor clínico de la exploración electrofisiológica ocular, electrorretinografía y potenciales evo- cados visuales, en los diferentes estadios evolutivos de las cataratas con relación a su pronóstico visual postoperatorio. Tesis Doctoral, Facultad de Medicina, Universidad Complutense de Madrid, Madrid, España.

Pontes A, LP Lazaro, C Dos Santos, F Dorea, JL Laus. 2004. Considerations about electroretinography in dogs. Cienc Rural 34, 323-328.

Qiu H, E Fujiwara, M Liu, BL Lam, DI Hamasaki. 2002. Evidence that a-wave latency of the electroretinogram is determined solely by photoreceptors. Jpn J Ophthalmol 46, 426-432.

Ropo A, P Ruusuvaara, K Setälä. 1992. Visual evoked potentials after retrobulbar or periocular anaesthesia. $\mathrm{Br} \mathrm{J}$ Ophthalmol 76, 541-544.

Ropstad EO, K Narfström. 2007. The obvious and the more hidden components of the electroretinogram. Eur J Companion Anim Pract 17, 290-296.

Rosolen SG, F Rigaudiere, P Lachapelle. 2002. A practical method to obtain reproducible binocular electroretinograms in dogs. Doc Ophthalmol 105, 93-103.

Safatle AMV, S Salomão, A Berezovsky, P Sacai, D Fantoni, K Yasbek, PSM Barros. 2005. Retinal degeneration in a Pit Bull dog: electroretinographic findings. Arch Vet Sci 10, 119-124.

Sato S, S Sugimoto, S Chiba. 1982. A procedure for recording electroretinogram and visual evoked potential in conscious dogs. J Pharmacol Meth 8, 173-181.

Sims MH, LJ Laratta, WJ Bubb, RV Morgan. 1989. Waveform analysis and reproducibility of visual-evoked potentials in dogs. Am J Vet Res 50, 1823-1828.

Sims MH. 1990. Partial masking of the canine electroretinogram by oscillatory potentials- to problem of frequency bandwidth. J Vet Intern Med 4, 40-42.

Steiss JE. 2003. Electrodiagnostic evaluation. In: Braund KG (ed). Clinical Neurology in Small Animals - Localization, Diagnosis and Treatment. $1^{\text {st }}$ ed. International Veterinary Information Service (IVIS), Ithaca, New York, USA, Pp 323-337.

Strain GM, RM Jackson, BL Tedford. 1990. Visual Evoked Potentials in the clinically normal dog. J Vet Intern Med 4, 222-225.

Strain GM, MS Claxton, JS Prescott-Mathews, DJ LaPhand. 1991. . Electroretinogram and Visual-evoked potential measurements in sheep. Can J Vet Res 55, 1-4.

Strain GM, RM Jackson, BL Tedford. 1991 ${ }^{\mathrm{b}}$. Postnatal development of the visual-evoked potential in dogs. Am J Vet Res 52, 231-235.

Uzuka Y, S Doi, M Tokuriki, H Matsumoto. 1989. The establishment of a clinical diagnostic method of the Visual Evoked Potentials (VEPs) in the cat: the effects of recording electrode positions, stimulus intensity and the level of anesthesia. Jpn J Vet Sci 51, 547-553.

Zaragoza P. 2011. Aplicaciones clínicas de la electrofisiología ocular. $1^{\mathrm{a}}$ ed. Sociedad Española de Oftalmología (SEO), Madrid, España. 
